\title{
HORMONAL ACTIONS IN THE PROTOZOAN STRESS: A REVIEW
}

\author{
GYÖRGY CSABA* \\ Department of Genetics, Cell and Immunobiology, Semmelweis University, \\ Budapest, Hungary
}

(Received: 23 September 2015; accepted: 22 October 2015)

\begin{abstract}
In the higher ranked animals the alteration of the environment can provoke a uniform reaction named general adaptation system (GAS), which is a manifestation of stress, caused by different stressors. During GAS certain organs show typical reactions and two members of the hormonal system are activated: epinephine and glucocorticoids. As the unicellular ciliate Tetrahymena also synthesize most of the mammalian-like hormones (except steroids), it can respond to stress by a hormonal reaction. The main differences, related to the mammalian GAS hormonal reaction are, that 1) in Tetrahymena the level of all of the hormones studied significantly elevates under the effect of heat, osmotic or chemical stress and 2) the single stress effect is durable. It is manifested at least to the 100th generations, which means that it is inherited epigenetically. Not only hormone synthesis but the receptorial hormone binding is also elevated, which means that the whole hormonal system is activated. The stress reaction (GAS) phylogenetically can be deduced to a unicellular (Protozoan) level however, prokaryotes - which are also stress-reactive - are using another mechanisms.
\end{abstract} binding

Keywords: Tetrahymena, stress hormones, prokaryotes, GAS, hormone

\section{Introduction}

Hans Selye published a short letter in Nature in 1936 in which he described a general reaction to different harms in mammals. The characteristic reaction was the enlargement of adrenal glands, the atrophy of thymus and lymphoid organs as well, as gastric erosion [1]. Later he observed the role of catecholamines (epinephrine, norepinephrine), glucocorticoids and the hypothalamo-pituitary system in this reaction. The whole system, which was participating in the reaction was named by him as general adaptation system (GAS). The phenomenon evolved by the harmful agents getting the name stress, while the provoking

*E-mail: csagyor@dgci.sote.hu 
agents were named stressors. According to Greenberg et al., "stress involves real or perceived changes within an organism in the environment that activate an organism's attempts to cope by means of evolutionarily ancient neural or endocrine mechanisms" [2]. Considering this, the stressor can be any chemical or physical agent, starvation, psychical alteration, etc. The organisms try to avoid the stress or eliminate the stressor. If it is not possible, the stress is manifesting, the hormonal reaction starts and the changes in the stress-touched organs are taking place. Stress is inevitable during life, however - as Selye declared later it is not only dangerous (distress) but it could be beneficial (eustress), and this latter is needed for the normal function of life [1]. However, distress can lead to the exhaustion of the organism and consequently to death.

Stress is a general reaction and also general, considering the species, as not only mammals, but lower ranked animals also have it. However, the endocrine participants are frequently other than in mammals. For example in insects the norepinephrine-like hormone is octopamine and an other molecule, called adipokinetic hormone, has the function of glucocorticoids in the reaction [3]. In worms insulin seems to be the hormone participating in stress reactions [4, 5]. The question is, whether in the simplest eukaryotic organisms, in Protozoa are there traces of the stress reaction or the general adaptation system, or in other words, can the stress (GAS) reaction be deduced to a unicellular level? For this aim the study of Tetrahymena seems to be the most suitable.

The unicellular ciliate Tetrahymena is not only a cell, but an organism, "the most highly developed protozoan, because it possesses specialized organelles that perform each of the cell functions" [6]. It also has - what is the most important from our point of view - a complete hormonal system [7, 8]. It synthesizes amino acid type (serotonin, histamine, epinephrine) and polypeptide (insulin, adrenocorticotropin, gonadotropin, endorphin) hormones and does not produce steroids (glucocorticoids), which have central role in the GAS of the mammals. It can also store and secrete the hormones produced and can influence the behavior and function of the Tetrahymena population by them, as the cells have membrane receptors for recognizing and binding and also deciphering the code of mammalian-like hormones [7, 8]. The message contained by the hormone is transmitted to cell organelles for responding to it, as the cells have signal transduction systems, similar to that of mammals (adenylate cyclase and guanylate cyclase system, Ca-calmodulin system, phosphoinositol system). A minute amount of hormones (e.g. 10-21 M) is enough for provoking response [9] and the cells can be imprinted by the first encounter with the hormone. The imprinting is hereditary, its effect is present after 1000 generations [10]. Tetrahymena can be stressed by heat or starvation and chemical substances, and the effect of stressors can be observed by different ways. The stress of Tetrahymena has a very broad 
spectrum of experiments, however, here only those will be mentioned, which are studied in the hormonal response to stress.

\section{Effect of stress to the hormonal system of Tetrahymena}

Tetrahymena is stress-sensitive and many factors can be used as stressors in laboratory experiments. For Tetrahymena pyriformis, which was used in the presently discussed experiments, $28{ }^{\circ} \mathrm{C}$ is optimal for maintenance. Higher or lower temperature stresses the cells. Also stressors are the starvation, especially if it is employed for a longer time and the osmotic changes, especially the higher salt concentrations. Formaldehyde and ethanol are also stressors. Short $(1 \mathrm{~h})$ treatment of the cells with different (higher than physiological: $20 \mathrm{mg} / \mathrm{ml} \mathrm{medium)}$ concentrations of the cells with $\mathrm{NaCl}$ or $\mathrm{KCl}$ as well as higher $\left(37^{\circ} \mathrm{C}\right)$ or lower $\left(4{ }^{\circ} \mathrm{C}\right)$ temperature for $1 \mathrm{~h}$ almost doubled the hormone production of cells and similar was the situation when high temperature $\left(37^{\circ} \mathrm{C}\right)$ or formaldehyde was used. However, $0.1 \%$ alcohol also significantly elevated the hormone levels [11].

The effect of stressors is durable. Heat stress $\left(37^{\circ} \mathrm{C}\right)$ for $1 \mathrm{~h}$ causes significant increase in the level of serotonin, histamine and triiodothyronine (T3) for two weeks, which later (after 8 weeks) decreases to control level or under it [12].

Short $(30 \mathrm{~min})$ starvation hardly influences the hormone content of cells (only serotonin elevated), however, long starvation $(24 \mathrm{~h})$ increased with $50 \%$ the production of beta-endorphin, adrenocorticotropin (ACTH), serotonin, histamine, insulin and T3 (all of the hormones are produced normally by Tetrahymena). After a short (30 min) re-feeding endorphin and histamine returned to the basal level, ACTH and histamine decreased but remained significantly higher than normal, and insulin and T3 remained at the starvation level [13, 14].

Other stressors also influenced the hormon synthesis of Tetrahymena. These were $\mathrm{CuSO}_{4}$, parachlorophenylalanine (PCPA, a tryptophan hydroxylase inhibitor). The level of ACTH, endorphin, serotonin, T3, histamine, insulin, epinephrine were significantly increased by them, however, histamine and T3 were less sensitive to these stressors. Insulin enhanced the hormone-level increasing effect of stressors [15].

Not only the hormone-production, but hormone binding was also stimulated by stress. Long ( $24 \mathrm{~h})$ starvation elevated the insulin binding of Tetrahymena and this was not changed immediately after short re-feeding $[14,16]$. Other stressors, as 5,10 , or $20 \mathrm{mg} / \mathrm{ml} \mathrm{NaCl}$ or $0.01 \%, 0.1 \%$ or $0.05 \%$ formaldehyde treatment as well as $37^{\circ} \mathrm{C}$ thermal stress elevated the insulin binding by Tetrahymena. However $4 \mathrm{~h}$ after osmotic stress the hormone binding decreased and after formaldehyde stress it was elevated. 


\section{Conclusions}

Tetrahymena synthesizes, stores and secretes any mammalian-like hormones, which were studied at all, except - in normal conditions - steroid hormones. These hormones also can bind to Tetrahymena and using transduction systems similar to those of mammals activate different functions. As the secretion of hormones takes place into the watery milieu of the producer cells, there is a possibility of influencing themselves as well, as the other cells of the population being in the neighbourhood of the producers. As very low (sometimes 10-21 M) concentration is enough for the activation of functions, the effectivity is very high. Considering these facts, this hormonal system is able to alarm the population of Tetrahymena.

The experiments, which were done for studying the stress reaction of Tetrahymena unanimously demonstrate the presence of a system, which reacts to harmful conditions (stressors) at this unicellular level. The hormonal system of Tetrahymena is mobilized under the pressure of stressors, maybe due to a hormonal network [6], hormone synthesis starts and hormone binding is also altered. As many hormones were investigated it is obvious that the reaction is general, all of the hormone levels (and hormone binding) were elevated. The hormone level increase is similar to that of GAS in mammals however, the activated hormone is not epinephrine (at first) and a glucocorticoid (later), as Tetrahymena does not have this latter and is not single, as each hormone of the cell are involved in the reaction. For explaining this there are two possibilities: 1) the Tetrahymena is not able to react with a separated hormone reaction in general or 2) each hormone studied are needed for defending the stress (distress). The second statement seems to be more likely, as in some other experiments hormone synthesis moved in two directions [8]. However, not only each hormone is mobilized, but each stressor - heat, osmotic alteration, chemicals - provoke the same reaction. This reaction includes - in addition to the enhanced hormone synthesis - the elevation of hormone binding capacity which helps the propagation of hormonal effects inside the population. If the hormones support the tolerability of stress, this complex activity of the hormonal system of Tetrahymena also helps the survival of the population.

Considering that the production of each hormones, produced by Tetrahymena is activated by the effect of stress in contrast to the elevation of norepinephrine and cortisol in mammals, it can be declared that this is a characteristic difference in the two levels of evolution. However, there are not negative data on the behaviour of other hormones in mammals during stress. Selye observed the effect of the two hormones mentioned and the systematic study of other hormones were neglected. If in the case of Tetrahymena only epinephrine would 
have been studied, it would be believed that identical hormones are participating in the reaction at the two levels. However, the comparison between the two philogenetic levels is uncertain at present.

How can the enhanced hormone production help the survival? The hormones produced by the Tetrahymena are influencing many life functions. Histamine and serotonin stimulates phagocytosis [17], as well as the protein binding capacity of the cell membrane [18] and by this, hormone reception. Epinephrine enhances phagocytosis [19] and glucose metabolism [20], triiodothyronine stimulates cell division [19], insulin influences sugar metabolism [21] and as a general effect, all of the hormones influence RNA synthesis [22]. As stressors offend the whole organism and the hormones influence basic life functions, the defence stimulated by hormones absolutely needed. These effects are not known in details, but the data which are at our disposal show the useful and needed effects of hormones.

The stressor-activated hormonal reaction is not restricted only to the Tetrahymena population which directly met the stressor, but inherited also to the progenies. Up to two weeks the hormone levels are higher than normal, which shows that at least 100 generations are touched by the single stress. For this time the cells are in an alarm-situation, preparing themselves to a further attack of stressors. This is understandable, as the individual life of Tetrahymena is relatively short, two or three hours, the transmission of experience is needed for the survival of the population. The phenomenon is similar to the hormonal imprinting, when the first encounter with a receptor-level acting molecule $[10,23]$ provokes the everlasting (studied up to 1000 generations) alteration of cell functions, however, after stress it is shorter.

As it was mentioned earlier, in higher ranked animals, as worms, insects, mammals there is a stress hormone, glucocorticoid, octopamine, insulin, the level of which is increasing under the pressure of stressors and activates the further reactions. Considering this, what would be the dominating stress hormone of Tetrahymena, if it is known that all hormones' levels are increasing during stress? It is very difficult to name one of the hormones however, insulin seems to be the most worth to the name. Insulin in Tetrahymena influences not only the sugar metabolism $[23,24]$, but many other important functions and it has a life saving property, when only few cells are present $[25,26]$. In the stress experiments it also had the most consequent behaviour. It seems to be the "primus inter pares" in the action against the effect of stress. However, it must be cautious to declare the outstanding role of insulin, as this hormone was studied the most thoroughly [23] when its effects were demonstrated and mammalian-like receptors were found [27-29] and there are very scarce data on the role of other hormones from this aspect. 
As Tetrahymena is living in a watery milieu, it can respond only to watersoluble molecules. This can explain why only amino acid and peptide hormones are participating in its stress response. At the same time, the receptor and transducer system of these hormones react more quickly and can develop response and this is advantageous in an alarm reaction.

It is not sure, that each Tetrahymena can tolerate the stress at all. There are data that an extremely high salt concentration destroys the cells and only a small part of the population can survive the stress caused by it [30]. These are the stress-tolerant cells, which give only $2 \%$ of the whole population. However, stress tolerance could be hereditary, and after the regrowth of the population the number of tolerant cells increases.

It is interesting that during starvation stress the protein synthesis significantly decreases, in contrast to the peptide hormone synthesis, which is enhanced. This also points to the rather important function of hormones in case of stress.

In higher ranked animals not only the hormonal reaction is characteristic to GAS, but alterations of different organs. How does Tetrahymena react to harmful agents or conditions in the frame of GAS? Cell volume significantly decreases in high $\mathrm{NaCl}$ containing medium and the cells, which tolerated the high level of sodium, contain more of it and develop a sodium regulatory system with an increase of extrusion mechanism [30]. If there is a cold stress, Tetrahymena is increasing fatty acid desaturase to maintain proper membrane fluidity $[31,32]$. These and other data show that in contrast to the generality of hormonal response, the other stress activated actions are not uniform and dependent on the stressor and the requirements for defence.

It seems to be clear that the general adaptation system is working in Tetrahymena. However, as organs characteristic to the higher ranked animals are not present at this low level of phylogeny, only the hormonal part of the system is manifested, adapted to the requirements of the unicellular life. This means that the hormonal basis of GAS can be found at the lowest level of eukaryotic phylogeny and the other components are added to it during the evolution.

An other question is, what is the situation in prokaryotes, which are in a lower level of evolution. In bacteria there is also a communication, which can be intra- and interkingdom. In the intrakingdom communication the most well known way is the quorum sensing, by which the bacteria can recognize the density of population and can regulate the cell growth [33-35]. The materials which are participating in it are known and they are not similar to the mammalian or Tetrahymena hormones. Although such hormones and the enzymes which needed for their synthesis $[36,37]$ were demonstrated in some bacteria, their role is not known. Also a signal transduction system is not known similar to that of which is present in Tetrahymena or mammals. However, some mammalian hor- 
mones can influence the growth, behaviour or toxin production of bacteria, and these hormones are stress-hormones in mammals [35]. This means that hoststress is influencing bacteria, the function of which is responsive to stress-hormones. Epinephrine, nor-epinephrine and dopamine can enhance the growth and toxin production of different bacteria, while decrease the immune functions of the hosts [35]. It can be concluded that prokaryotes also have a language for intrakingdom and interkingdom communication [35], but the vertebrate endocrine system cannot be deduced to it. It seems to be right to declare in our present knowledge that the protozoan level is phylogenetically the lowest in which the signs of the endocrine system is present. However, it must be considered that Tetrahymena is a very high-level product of the protozoan phylogeny and at this level also could have been an evolution which produced this system. This also produced the hormonal stress reaction, which became more sophisticated during the later steps of evolution.

\section{References}

1. Szabo, S., Tache, Y., Somogyi, A.: The legacy of Hans Selye and the origins of stress research: A retrospective 75 years after his landmark brief "Letter" to the Editor of Nature. Stress 15, 472-478 (2012).

2. Greenberg, N., Carr, J. A., Summers, C. H.: Causes and consequences of stress. Integr Comp Biol 42, 508-516 (2002).

3. Schulte, P. M.: What is environmental stress? Insights from fish living in a vairable environment. J Exp Biol 217, 23-34 (2014).

4. Mondoux, M. A., Love, D. C., Ghosh, S. K., Fukushige, T., Bond, M., Weerasinghe, G. R., Hanover, J. D., Krause, M. W.: O-linked - N-acetylglucosamine cycling and insulin signaling are required for glucose stress response in Caenorhabditis elegans. Genetics 188, 369-382 (2011).

5. Oláhová, M., Veal, E. A.: A peroxiredoxin, PRDX 2 required for insulin secretion and insulin/IIS-dependent regulation of stress dependence and longevity. Aging Cell 14, 558568 (2015).

6. Zhang, S., Ling, Z., Wang, S., Nozawa, Y., Umeki, S.: Tetrahymena cell culture. Wiley Online Libary, 2010.

7. Csaba, G.: The unicellular Tetrahymena as a model cell for receptor research. Int Rev Cytol 95, 327-377 (1985).

8. Csaba, G.: The hormonal system of the unicellular Tetrahymena: A review with evolutionary aspects. Acta Microbiol Immunol Hung 59, 131-156 (2012).

9. Csaba, G., Kovács, P., Pállinger, É.: How does the unicellular Tetrahymena utilize the hormones that it produces? Paying a visit to the realm of atto- and zeptomolar concentrations. Cell Tissue Res 327, 199-203 (2007).

10. Köhidai, L., Lajkó, E., Pállinger, É., Csaba, G.: Verification of epigenetic inheritance in a unicellular model system: Multigenerational effects of hormonal imprinting. Cell Biol Int 36, 951-959 (2012). 
11. Csaba, G., Pállinger, É.: A general response to stressors by the unicellular Tetrahymena: effect of stress on the hormone levels. Cell Biochem Funct 26, 797-800 (2009).

12. Lajkó, E., Pállinger, É., Csaba, G.: Durable effect of heat-stress on the hormone production of Tetrahymena. Effect of insulin on the consequence of stress. Acta Microbiol Immunol Hung 59, 249-256 (2012).

13. Csaba, G., Kovács, P., Pállinger, É.: Increased hormone levels in Tetrahymena after longlasting starvation. Cell Biol Int 31, 924-928 (2007).

14. Csaba, G., Kovács, P.: Comparison of the insulin binding, uptake and endogeneous insulin content in long- and short-term starvation in Tetrahymena. Cell Biochem Funct 26, 64-69 (2008).

15. Csaba, G., Pállinger, É.: How applicable is the general adaptation syndrome to the unicellular Tetrahymena? Cell Biochem Funct 27, 12-15 (2009).

16. Csaba, G., Pállinger, É.: Effect of stress and stress hormones on the hormone (insulin) binding of Tetrahymena. Cell Biochem Funct 27, 448-451 (2009).

17. Csaba, G., Lantos, T.: Effect of hormones on Protozoa. Studies on the phagocytotic effect of histamine, 5-hydroxytryptamine and indoleacetic acid in Tetrahymena pyriformis. Cytobiologie 7, 361-365 (1973).

18. Kovács, P., Csaba, G., Csöregh, É.: Influence of endocytosis stimulating hormones on the protein binding capacity of the cell membrane. Acta Physiol Hung 61, 213-216 (1983).

19. Csaba, G.: Biogenic amines at a low level of evolution: Production, functions and regulation in the unicellular Tetrahymena. Acta Microbiol Immunol Hung 62, 93-108 (2015).

20. Csaba, G., Kovács, P.: Complex cytophotometric analysis of the hormone-induced alterations in the glucose utilisation of Tetrahymena. Endokrinologie 73, 116-119 (1979).

21. Csaba, G., Lantos, T.: Effect of insulin on the glucose uptake of protozoa. Experientia 31, 1097-1098 (1975).

22. Csaba, G., Ubornyák, L.: Effect of hormones on the RNA synthesis of Tetrahymena pyriformis. Comp Biochem Physiol C 68, 251-253 (1981).

23. Csaba, G.: Insulin at a unicellular eukaryote level. Cell Biol Int 37, 267-275 (2013).

24. Köhidai, L., Csaba G.: Effects of insulin and histamine in themselves and in combination on the glucose metabolism of Tetrahymena. Acta Biol Hung 36, 281-285 (1983).

25. Christensen, S. T.: Insulin rescues the unicellular eukaryote Tetrahymena from dying in a complete synthetic nutrint medium. Cell Biol Int 17, 833-837 (1993).

26. Christensen, S. T., Kemp, K., Rasmussen, L.: Insulin produces a biphasic response in Tetrahymena thermophila by stimulating cell survival activity of proliferation in two separate concentration intervals. Cell Biol Int 20, 437-444 (1996).

27. Christopher, G. K., Sundermann, C. A.: Conventional and confocal microscopic studies of insulin receptor induction in Tetrahymena pyriformis. Exp Cell Res 201, 477-484 (1992).

28. Christopher, G. K., Sundermann, C. A.: Isolation and partial characterisation of the insulin binding sites of Tetrahymena pyriformis. Biochem Biophys Res Com 212, 515-523 (1995).

29. Christensen, S. T., Guerra, C. F., Wheatley, D. N., Satir, P.: Insulin receptor-like proteins in Tetrahymena ciliary membranes. Curr Biol 13, R50-52 (2003).

30. Dunham, P. B.: The adaptation of Tetrahymena to a high $\mathrm{NaCl}$ environment. Biol Bull 126, 373-390 (1964).

31. Nozawa, Y.: Adaptive regulation of membrane lipids and fluidity during thermal acclimation in Tetrahymena. Proc Jpn Acad Sci B 87, 450-462 (2011). 
32. Nakashima, S., Nozawa, Y.: Stress responsive gene expression in Tetrahymena. Acta Biol Hung 50, 413-424 (1999).

33. Boor, K. J.: Bacterial stress responses: What does not kill them, can make them stronger. PloS Biol 4, e23 (2006).

34. Freestone, P.: Communication betwen bacteria and their hosts. Scientifica 2013, 361073 (2013).

35. Hughes, D. T., Sperandio, V.: Inter-kingdom signaling: Communication between bacteria and their hosts. Nat Rev Microbiol 6, 111-120 (2008).

36. Takeda, N., Sugiyama, K.: Metabolism of biogenic monoamines in the ciliated protozoan, Tetrahymena pyriformis. Comp Biochem Physiol C 106, 63-70 (1993).

37. Roth, J., Le Roith, D., Schiloach, R., Rosenzweig, J. L, Lesniak, M. A, Havrankova, J.: The evolutionary origin of hormones, neurotransmitters and other extracelluler chemical messengers, implications for mammalian biology. N Engl J Med 366, 523-527 (1982). 